\title{
SIMPLIFICATION AND REDUCTION OF THE SANDERS-KOITER LINEAR SHELL EQUATIONS FOR VARIOUS MIDSURFACE GEOMETRIES*
}

\author{
BY \\ JAMES G. SIMMONDS \\ University of Virginia
}

\begin{abstract}
It is shown that the twelve linear field equations of the Sanders-Koiter first approximation shell theory, when specialized to shells with midsurfaces of constant mean curvature, can be replaced by eight equations of particularly simple form. This is accomplished by adding certain negligibly small terms to the conventional uncoupled stress-strain relations. On the basis of order of magnitude estimates, it is argued that these equations are actually adequate for shells of arbitrary geometry. For shells of nonzero Gaussian curvature, the simplified field equations are reduced to four coupled scalar equations. For catenoidal and helicoidal shells, these equations are further reduced to two coupled fourth-order equations. Various special forms of these equations are shown to agree with results obtained by Lardner, Reissner, and Wan for shallow shells, shells of revolution undergoing axisymmetric and lateral deformation, and helicoidal shells undergoing axisymmetric deformation.
\end{abstract}

1. Introduction. This paper is concerned with simplifying, without any essential loss of accuracy, the linear field equations of the Sanders-Koiter first approximation shell theory [1], [2], [3]. These field equations consist of the twelve equilibrium, compatibility, and stress-strain relations for the components of a set of symmetric stress and strain tensors. We proceed in two stages.

First, we show for shells of constant mean curvature $H$ that the field equations take a particularly simple form, provided only that certain small, explicitly computable terms are added to the conventional, uncoupled stress-strain relations. The modified stressstrain relations are as accurate as the unmodified ones, since the differences between the two involve terms of the same order of magnitude as the inherent errors in the unmodified stress-strain relations. Two of the field equations simplify still further if $H=0$. Some order of magnitude arguments are then advanced to conclude that the simplified field equations for $H=0$ are sufficiently accurate for shells of arbitrary geometry.

In the second stage we begin with the observation that the simplified equilibrium and compatibility equations can each be expressed as the divergence of a space vector. Using an idea due to Libai [4], which was further developed by the author [5] in reducing a simplified set of field equations proposed by Sanders [6], we reduce the vector equations for shells of nonzero Gaussian curvature $(K \neq 0)$ to four coupled scalar equations. If $I I=0$, these equations simplif $y$ considerably and, for catenoidal and helicoidal shells,

* Received September 8, 1969. This work was supported by the National Science Foundation under grant GP-7759. 
they are further reduced to two coupled fourth-order equations. Several special cases of these equations are shown to agree with earlier work of Reissner and his students.

There have been several notable and valuable attempts in the literature to reduce the general equations of shell theory. Novozhilov [7] showed that if Poisson's ratio is zero, the equilibrium and compatibility equations can be pair-wise combined by the introduction of complex-valued quantities. He also attempted to argue, on the basis of order of magnitude considerations, that his simplified equations would still be sufficiently accurate if Poisson's ratio were not zero. However, Koiter pointed out [8] that Novozhilov's equations did not adequately describe the axisymmetric deformation of a helicoidal shell. Furthermore, our results show that Novozhilov's single complex, constant-coefficient equation for catenoidal shells is generally inadequate (unfortunately, our more accurate equations have variable coefficients and cannot be combined into a single complex equation).

In a recent paper [6] Sanders attempted to eliminate the deficiencies in Novozhilov's equations. In an ingenious analysis, he showed that by adding certain terms to the stress-strain relations, three simplified equilibrium-compatibility equations could be obtained for a symmetric, complex-valued tensor of second order. He then showed how these equations could be reduced to a single fourth-order equation for a complex-valued scalar for a variety of shell geometries. The success of Sanders' method rests upon showing that the terms added to the stress-strain relations are of a certain order of magnitude. For cylindrical shells of arbitrary cross section, he was able to determine these terms explicitly. However, in an addendum to his paper, he stated that for a helicoidal shell undergoing axisymmetric deformation, it is impossible to choose the required additional terms in the stress-strain relations to be of the right order of magnitude. The fact that we are unable to combine our reduced equations for helicoidal shells into a single complex-valued equation, even for the special case of axisymmetric deformation, corroborates Sanders' finding, because it shows that there is no fourth-order "characteristic equation" for the helicoidal shell, as Sanders' reduced equations would imply were they universally valid.

The work of two other writers should be mentioned. Goldenveizer [9], starting from the stress-strain relations with the stresses and strains expressed in terms of three stress functions and three displacement components, respectively, obtained a determinantal equation for a complex-valued function whose real and imaginary parts are the midsurface normal displacement and one of the stress functions. His final equations, being enormously complicated and of sixth rather than fourth order, seem of little practical value. In a paper just published [10], F. Y. M. Wan considers complete shells of revolution under arbitrary, self-equilibrated loads. Using Fourier series, he obtains a set of equations for the Fourier components of the unknowns. These he reduces to two coupled fourth-order equations. Whether this procedure will work for shells of revolution of arbitrary shape when Fourier decomposition is not possible, and whether, if it does, the resulting partial differential equations will be of no higher order than the unreduced equations is an open question.

2. Simplification of the governing equation. The field equations of the SandersKoiter linear shell theory [1], [2], [3] consist of three exact equilibrium equations ${ }^{1}$

$$
\left.\left(N^{\alpha \beta}+{ }_{2}^{1} \epsilon^{\alpha \beta} B_{\lambda \mu} M^{i \mu \mu}\right)\right|_{\alpha}+\left.b_{\alpha}^{\beta} M^{\alpha \gamma}\right|_{\gamma}=0,
$$

${ }^{1}$ For simplicity, we assume throughout that there are no surface loads. 


$$
-\left.M^{\alpha \beta}\right|_{\alpha \beta}+b_{\alpha \beta} N^{\alpha \beta}=0,
$$

three exact compatibility equations

$$
\begin{gathered}
\left.\left(\bar{K}^{\alpha \beta}-\frac{1}{2} \epsilon^{\alpha \beta} B_{\lambda_{\mu}} \bar{E}^{\lambda \mu}\right)\right|_{\alpha}-\left.b_{\alpha}^{\beta} \bar{E}^{\alpha \gamma}\right|_{\gamma}=0, \\
\left.\bar{E}^{\alpha \beta}\right|_{\alpha \beta}+b_{\alpha \beta} \bar{K}^{\alpha \beta}=0,
\end{gathered}
$$

plus six isotropic stress-strain relations which are usually taken in the uncoupled form

$$
\begin{aligned}
M^{\alpha \beta} & =D\left[a^{\alpha \beta} K_{\lambda}^{\lambda}-(1-\mu) \bar{K}^{\alpha \beta}\right], \\
\bar{E}^{\alpha \beta} & =A\left[a^{\alpha \beta} N_{\lambda}^{\lambda}-(1+\nu) N^{\alpha \beta}\right] .
\end{aligned}
$$

These field equations exhibit the material-static-geometric analogy:

$$
\begin{aligned}
N^{\alpha \beta} & \leftrightarrow \bar{K}^{\alpha \beta} & M^{\alpha \beta} & \leftrightarrow-\bar{E}^{\alpha \beta} \\
A & \leftrightarrow-D & \mu & \leftrightarrow-\nu .
\end{aligned}
$$

In (2.1)-(2.6), the following quantities and notation have been introduced: $N^{\alpha \beta}$ and $M^{\alpha \beta}$ are modified symmetric stress resultants and couples related to the conventional unsymmetric stress resultants and couples $\widetilde{N}^{\alpha \beta}$ and $\widetilde{M}^{\alpha \beta}$ by the equations

$$
\begin{aligned}
& N^{\alpha \beta}=\tilde{N}^{\alpha \beta}-b_{\gamma}^{\beta} \tilde{M}^{\gamma \alpha}+\frac{1}{2}\left(b_{\gamma}^{\alpha} M^{\gamma \beta}+b_{\gamma}^{\beta} M^{\alpha \gamma}\right), \\
& M^{\alpha \beta}=\frac{1}{2}\left(\tilde{M}^{\alpha \beta}+\tilde{M}^{\beta \alpha}\right) .
\end{aligned}
$$

$a_{\alpha \beta}, b_{\alpha \beta}$, and $\epsilon_{\alpha \beta}$ are, respectively, the metric, curvature, and permutation tensor of the shell midsurface. $B_{\alpha \beta}$, introduced by Sanders [6], is defined by

$$
B_{\alpha \beta}=\frac{1}{2}\left(\epsilon_{\alpha \gamma} b_{\beta}^{\gamma}+\epsilon_{\beta \gamma} b_{\alpha}^{\gamma}\right) .
$$

This tensor is independent of the three standard surface tensors in the sense that

$$
a_{\alpha \beta} B^{\alpha \beta}=b_{\alpha \beta} B^{\alpha \beta}=\epsilon_{\alpha \beta} B^{\alpha \beta}=0 .
$$

To minimize the number of minus signs in the various equations to follow, we have used the conventional definition of $b_{\alpha \beta}$ and adopted Koiter's definition [2] of $M^{\alpha \beta}$ and $K^{\alpha \beta}$. Budiansky and Sanders [3] define these three tensors with the opposite sign. Either way, the field equations are identical in the form and sign.

All indices are raised and lowered using $a_{\alpha \beta}$, and a vertical bar denotes covariant differentiation with respect to a set of arbitrary surface coordinates $\theta^{\alpha}, \alpha=1,2$. The "bar" notation, defined for any tensor $A^{\alpha \beta}$ by

$$
\bar{A}^{\alpha \beta} \equiv \epsilon^{\alpha \lambda} \epsilon^{\beta \mu} A_{\lambda \mu}=a^{\alpha \beta} A_{\lambda}^{\lambda}-A^{\alpha \beta} \text { etc. }
$$

was devised by Libai [4] and Sanders [6] and considerably condenses a number of shell equations.

In the stress-strain relations, $D$ is the bending stiffness and $\mu$ is Poisson's ratio of bending; $A$ and $\nu$ are the analogous stretching factors. It is customary, but not necessary, to tinise

$$
D=\frac{E h^{3}}{12\left(1-\nu^{2}\right)}, \quad A=\frac{1}{E h}, \quad \mu=\nu,
$$

where $E$ is Young's modulus and $h$ is the shell thickness. 
The simplifications considered in this section are restricted to shells with midsurfaces of constant mean curvature $H=\frac{1}{2} b_{\alpha}^{\alpha}$. We began by replacing (2.5) and (2.6) by the modified stress-strain relations

$$
\begin{aligned}
M^{\alpha \beta} & =D\left[a^{\alpha \beta} K_{\lambda}^{\lambda}-(1-\mu) \bar{K}_{*}^{\alpha \beta}\right], \\
\bar{E}^{\alpha \beta} & =A\left[a^{\alpha \beta} N_{\lambda}^{\lambda}-(1+\nu){N_{*}^{\alpha \beta}}^{\alpha \beta} .\right.
\end{aligned}
$$

We shall show, by explicit computation, that $K_{*}^{\alpha \beta}$ and $N_{*}^{\alpha \beta}$ may be chosen so that the field equations take a particularly simple form and such that

$$
\begin{aligned}
& K_{*}^{\alpha \beta}=K^{\alpha \beta}+O(E / R), \\
& N_{*}^{\alpha \beta}=N^{\alpha \beta}+O(M / R),
\end{aligned}
$$

where $E$ and $M$ denote, respectively, the maximum absolute value of the extensional strain and the stress couple and $R$ denotes the minimum principle midsurface radius. The order of magnitude relations (2.16) and (2.17) justify the use of (2.14) and (2.15), because they show that the modified and unmodified stress-strain relations differ by terms which are precisely of the same order of magnitude as the errors unavoidably contained in the stress-strain relations of any first approximation shell theory [2].

Inserting (2.14) and (2.15) into (2.1) to (2.4), noting (2.12), and observing that $\left.b^{\alpha \beta}\right|_{\alpha}=\left.b_{\alpha}^{\alpha}\right|^{\beta}=0$ implies

we get

$$
\begin{aligned}
\left.b_{\gamma}^{\beta} a^{\alpha \gamma} K_{\lambda}^{\lambda}\right|_{\alpha} & =\left.\left(b^{\alpha \beta} K_{\lambda}^{\lambda}\right)\right|_{\alpha}-\left.b^{\alpha \beta}\right|_{\alpha} K_{\lambda}^{\lambda} \\
& =\left.\left(b^{\alpha \beta} K_{\lambda}^{\lambda}\right)\right|_{\alpha},
\end{aligned}
$$

$$
\begin{aligned}
{\left.\left[N^{\alpha \beta}+D b^{\alpha \beta} K_{\lambda}^{\lambda}-\frac{1}{2} D(1-\mu) \epsilon^{\alpha \beta} B_{\lambda \mu} \bar{K}_{*}^{\lambda \mu}\right]\right|_{\alpha}-\left.D(1-\mu) b_{\gamma}^{\beta} \bar{K}_{*}^{\alpha \gamma}\right|_{\alpha}=0, } \\
-D\left[\nabla^{2} K_{\lambda}^{\lambda}-\left.(1-\mu) \bar{K}_{*}^{\alpha \beta}\right|_{\alpha \beta}\right]+b_{\alpha \beta} N^{\alpha \beta}=0, \\
{\left.\left[\bar{K}^{\alpha \beta}-A b^{\alpha \beta} N_{\lambda}^{\lambda}+\frac{1}{2} A(1+\nu) \epsilon^{\alpha \beta} B_{\lambda \mu} N_{*}^{\lambda \mu}\right]\right|_{\alpha}+\left.A(1+\nu) b_{\gamma}^{\beta} N_{*}^{\alpha \gamma}\right|_{\alpha}=0, } \\
A\left[\nabla^{2} N_{\lambda}^{\lambda}-\left.(1+\nu) N_{*}^{\alpha \beta}\right|_{\alpha \beta}\right]+b_{\alpha \beta} \bar{K}^{\alpha \beta}=0 .
\end{aligned}
$$

Except for spherical and circular cylindrical shells, ${ }^{2}$ it is impossible to choose $K_{*}^{\alpha \beta}$ and $N^{\alpha \beta}$ so that $\left.\bar{K}_{*}^{\alpha \beta}\right|_{\alpha}=\left.N_{*}^{\alpha \beta}\right|_{\alpha}=0$. However, let us assume that we can find scalars $\tilde{K}^{*}$ and $\tilde{N}$ such that

$$
\left.\bar{K}_{*}^{\alpha \beta}\right|_{\alpha}=\left.\epsilon^{\alpha \beta} \tilde{K}\right|_{\alpha} \quad \text { and }\left.\quad N_{*}^{\alpha \beta}\right|_{\alpha}=\left.\epsilon^{\alpha \beta} \tilde{N}\right|_{\alpha} .
$$

It then follows that

and

$$
\left.\tilde{K}_{*}^{\alpha \beta}\right|_{\alpha \beta}=0
$$

$$
\left.b_{\gamma}^{\beta} \bar{K}_{*}^{\alpha \gamma}\right|_{\alpha}=\left.\left(\epsilon^{\alpha \gamma} b_{\gamma}^{\beta} \tilde{K}\right)\right|_{\alpha}
$$

by virtue of the Codazzi equation $\left.\epsilon^{\alpha \gamma} b_{\gamma}^{\beta}\right|_{\alpha}=0$. Now use (2.10) to write

$$
\begin{aligned}
\epsilon^{\alpha \gamma} b_{\gamma}^{\beta} & =\frac{1}{2}\left(\epsilon^{\alpha \gamma} b_{\gamma}^{\beta}+\epsilon^{\beta \gamma} b_{\gamma}^{\alpha}\right)+\frac{1}{2}\left(\epsilon^{\alpha \gamma} b_{\gamma}^{\beta}-\epsilon^{\beta \gamma} b_{\gamma}^{\alpha}\right) \\
& =B^{\alpha \beta}+\epsilon^{\alpha \beta} I I .
\end{aligned}
$$

2 For these two special shells, severil alternate methods of simplification are available [6], [11], [12], [13]. 
With these relations and the analogous ones for $N_{*}^{\alpha \beta}$, we may put (2.19)-(2.22) into the form

$$
\begin{gathered}
\left.\left\{N^{\alpha \beta}+D b^{\alpha \beta} K_{\lambda}^{\lambda}-D(1-\mu)\left[B^{\alpha \beta} \tilde{K}+\frac{1}{2} \epsilon^{\alpha \beta}\left(B_{\lambda \mu} \bar{K}_{*}^{\lambda \mu}+2 H \tilde{K}\right)\right]\right\}\right|_{\alpha}=0 \\
-D \nabla^{2} K_{\lambda}^{\lambda}+b_{\alpha \beta} N^{\alpha \beta}=0 \\
\left.\left\{\bar{K}^{\alpha \beta}-A b^{\alpha \beta} N_{\lambda}^{\lambda}+A(1+\nu)\left[B^{\alpha \beta} \tilde{N}+\frac{1}{2} \epsilon^{\alpha \beta}\left(B_{\lambda \mu} N_{*}^{\lambda \mu}+2 H \tilde{N}\right)\right]\right\}\right|_{\alpha}=0 \\
A \nabla^{2} N_{\lambda}^{\lambda}+b_{\alpha \beta} \bar{K}^{\alpha \beta}=0 .
\end{gathered}
$$

It is now seen that if we choose

$$
\begin{aligned}
N_{*}^{\alpha \beta} & =N^{\alpha \beta}+D b^{\alpha \beta} K_{\lambda}^{\lambda}-D(1-\mu) B^{\alpha \beta} \tilde{K}, \\
\bar{K}_{*}^{\alpha \beta} & =\bar{K}^{\alpha \beta}-A b^{\alpha \beta} N_{\lambda}^{\lambda}+A(1+\nu) B^{\alpha \beta} \tilde{N}, \\
\tilde{N} & =\frac{1}{2} D(1-\mu)\left(B_{\lambda \mu} \bar{K}_{*}^{\lambda \mu}+2 H \tilde{K}\right), \\
\tilde{K} & =-\frac{1}{2} A(1+\nu)\left(B_{\lambda \mu} N_{*}^{\lambda \mu}+2 H \tilde{N}\right),
\end{aligned}
$$

the assumptions embodied in (2.16), (2.17), and (2.23) will all be fulfilled.

It is a straightforward matter, by eliminating $\tilde{K}$ and $\tilde{N}$ between the above four equations, to express $N_{*}^{\alpha \beta}$ and $\bar{K}_{*}^{\alpha \beta}$ explicitly in terms of $N^{\alpha \beta}$ and $\bar{K}^{\alpha \beta}$. Fortunately, we do not need these results to proceed further.

Finally, we introduce the two unsymmetric tensors

$$
\begin{aligned}
S^{\alpha \beta} & =N^{\alpha \beta}-D(1-\mu)\left[B^{\alpha \beta} \tilde{K}+\frac{1}{2} \epsilon^{\alpha \beta}\left(B_{\lambda \mu} \bar{K}_{*}^{\lambda \mu}+2 H \tilde{K}\right)\right] \\
& =N_{*}^{\alpha \beta}-D b^{\alpha \beta} K_{\lambda}^{\lambda}-\frac{1}{2} D(1-\mu) \epsilon^{\alpha \beta}\left(B_{\lambda \mu} \bar{K}_{*}^{\lambda \mu}+2 H \tilde{K}\right)
\end{aligned}
$$

and

$$
\begin{aligned}
\bar{T}^{\alpha \beta} & =\bar{K}^{\alpha \beta}+A(1+\nu)\left[B^{\alpha \beta} \tilde{N}+\frac{1}{2} \epsilon^{\alpha \beta}\left(B_{\lambda \mu} N_{*}^{\lambda \mu}+2 H \tilde{N}\right)\right. \\
& =\bar{K}_{*}^{\alpha \beta}+A b^{\alpha \beta} N_{\lambda}^{\lambda}+\frac{1}{2} A(1+\nu) \epsilon^{\alpha \beta}\left(B_{\lambda \mu} N_{*}^{\lambda \mu}+2 H \tilde{N}\right) .
\end{aligned}
$$

In view of (2.11), it follows that

$$
S \equiv S_{\alpha}^{\alpha}=N_{\alpha}^{\alpha}, \quad b_{\alpha \beta} S^{\alpha \beta}=b_{\alpha \beta} N^{\alpha \beta} \quad B_{\alpha \beta} S^{\alpha \beta}=B_{\alpha \beta} N_{*}^{\alpha \beta}
$$

with an analogous set of relations involving $\bar{T}^{\alpha \beta}$. Thus the field equations (2.27)-(2.30) take the form

$$
\begin{aligned}
\left.S^{\alpha \beta}\right|_{\alpha}+\left.D b_{\alpha}^{\beta} T\right|^{\alpha} & =0, \\
-D \nabla^{2} T+b_{\alpha \beta} S^{\alpha \beta} & =0, \\
\left.\bar{T}^{\alpha \beta}\right|_{\alpha}-\left.A b_{\alpha}^{\beta} S\right|^{\alpha} & =0, \\
A \nabla^{2} S+b_{\alpha \beta} \bar{T}^{\alpha \beta} & =0 .
\end{aligned}
$$

Note that (2.38)--(2.41) can be put into the concise vector form

$$
\left.S^{\alpha}\right|_{\alpha}=0,\left.\quad T^{\alpha}\right|_{\alpha}=0,
$$

where

$$
\begin{aligned}
& \boldsymbol{S}^{\alpha}=S^{\alpha \beta} \boldsymbol{a}_{\beta}-\left.D T\right|^{\alpha} n, \\
& \boldsymbol{T}^{\alpha}=\bar{T}^{\alpha \beta} \boldsymbol{a}_{\beta}+\left.A S\right|^{\alpha} n .
\end{aligned}
$$


Here $\boldsymbol{a}_{\beta}$ and $\boldsymbol{n}$ are the covariant base vectors and unit normal vector to the undeformed midsurface, which satisfy the Gauss-Weingarten equations

$$
a_{\alpha \mid \beta}=b_{\alpha \beta} n,\left.\quad n\right|_{\alpha}=-b_{\alpha}^{\beta} a_{\beta} .
$$

The six simplified field equations (2.38)-(2.41), involve eight unknowns. The following two additional conditions follow from (2.35), (2.36) and the last of (2.37):

$$
\begin{aligned}
& \epsilon_{\alpha \beta} S^{\alpha \beta}=-D(1-\mu)\left(B_{\lambda_{\mu}} \bar{T}^{\lambda \mu}+2 H \tilde{K}\right), \\
& \epsilon_{\alpha \beta} \bar{T}^{\alpha \beta}=A(1+\nu)\left(B_{\lambda \mu} S^{\lambda \mu}+2 H \tilde{N}\right) .
\end{aligned}
$$

By the last of (2.37), (2.33) and (2.34) can now be written

$$
\begin{aligned}
& \tilde{N}=\frac{1}{2} D(1-\mu)\left(B_{\lambda \mu} \bar{T}^{\lambda \mu}+2 H \tilde{K}\right), \\
& \tilde{K}=-\frac{1}{2} A(1+\nu)\left(B_{\lambda_{\mu}} S^{\lambda \mu}+2 H \tilde{N}\right) .
\end{aligned}
$$

If these two equations are solved for $\tilde{N}$ and $\tilde{K}$ and the results inserted into (2.47) and (2.48), it follows that

$$
\begin{aligned}
& \epsilon_{\alpha \beta} S^{\alpha \beta}=-D(1-\mu)\left(1+k^{2}\right)^{-1} B_{\lambda \mu}\left[\bar{T}^{\lambda \mu}-H A(1+\nu) S^{\lambda \mu}\right], \\
& \epsilon_{\alpha \lambda} \bar{T}^{\alpha \beta}=A(1+\nu)\left(1+k^{2}\right)^{-1} B_{\lambda \mu}\left[S^{\lambda \mu}+H D(1-\mu) \bar{T}^{\lambda \mu}\right],
\end{aligned}
$$

where

$$
k^{2}=H^{2} A D(1+\nu)(1-\mu)=O\left(h^{2} / R^{2}\right) .
$$

For shells of zero mean curvature, (2.51) and (2.52) reduce to

$$
\begin{aligned}
& \epsilon_{\alpha \beta} S^{\alpha \beta}=-D(1-\mu) B_{\lambda \mu} \bar{T}^{\lambda \mu}, \\
& \epsilon_{\alpha \beta} \bar{T}^{\alpha \beta}=A(1+\nu) B_{\lambda \mu} S^{\lambda \mu} .
\end{aligned}
$$

3. The adequacy of the simplified field equations for shells of arbitrary geometry. The aim of the preceding section was to indicate the extent of the simplifications possible for shells of constant mean curvature if one was constrained to produce a definite set of coupled stress-strain relations such that, starting with these, one could arrive at a set of simplified field equations without neglecting any terms whatsoever. Such a program led to particularly simple results when $H=0$.

In this section, we wish to show that by taking a somewhat more flexible (and hence less rigorous) approach, we may conclude that for shells of arbitrary midsurface geometry, the simplified field equations (2.38)-(2.41), (2.54) and (2.55) are as accurate as the full, unreduced Sanders-Koiter field equations.

Assume that in (2.1) and (2.2) we have used the conventional, uncoupled stressstrain relations (2.5) to express $M^{\alpha \beta}$ in terms of $K^{\alpha \beta}$. The resulting equations read

$$
\begin{gathered}
{\left.\left[N^{\alpha \beta}-\frac{1}{2} D(1-\mu) \epsilon^{\alpha \beta} B_{\lambda \mu} \bar{K}^{\lambda \mu}\right]\right|_{\alpha}+\left.D b^{\beta \alpha} K_{\lambda}^{\lambda}\right|_{\alpha}-\underline{\left.D(1-\mu) b_{\gamma}^{\beta} \bar{K}^{\alpha \gamma}\right]_{\alpha}=0,}} \\
{\left[D-\nabla^{2} K_{\lambda}^{\lambda}-\left.(1-\mu) \bar{K}^{\alpha \beta}\right|_{\alpha \beta}\right]+b_{\alpha \beta} N^{\alpha \beta}=0 .}
\end{gathered}
$$

From (2.3),

$$
\left.\bar{K}^{\alpha \beta}\right|_{\beta}=O(E / R L),
$$


where $L$ denotes the "characteristic wavelength" of the midsurface deformation pattern [2]. Now when dealing with the stress-strain relations (2.5) (and only then) we are free to add terms of the order $E / R$ to the bending strain tensor. Terms of this type lead to errors in the underlined terms in (3.1) and (3.2) of order $(D E / R L)$ and $\left(D E / R L^{2}\right)$, respectively. Thus, it is consistent to neglect the right-hand side of (3.3) when this relation is inserted into (3.1) and (3.2). It then follows that

$$
\begin{gathered}
{\left.\left[N^{\alpha \beta}-\frac{1}{2} D(1-\mu) \epsilon^{\alpha \beta} B_{\lambda \mu} \bar{K}^{\lambda \mu}\right]\right|_{\alpha}+\left.D b^{\alpha \beta} K_{\lambda}^{\lambda}\right|_{\alpha}=0,} \\
-D \nabla^{2} K_{\lambda}^{\lambda}+b_{\alpha \beta} N^{\alpha \beta}=0 .
\end{gathered}
$$

A strictly analogous set of arguments applied to the compatibility equations (2.3) and (2.4) and the stress-strain relations (2.6) leads to the simplified equations

$$
\begin{gathered}
{\left.\left[\bar{K}^{\alpha \beta}+\frac{1}{2} A(1+\nu) \epsilon^{\alpha \beta} B_{\lambda \mu} N^{\lambda \mu}\right]\right|_{\alpha}-\left.A b^{\alpha \beta} N_{\lambda}^{\lambda}\right|_{\alpha}=0,} \\
A \nabla^{2} N_{\lambda}^{\lambda}+b_{\alpha \beta} \bar{K}^{\alpha \beta}=0 .
\end{gathered}
$$

Thus by now defining

$$
\begin{aligned}
& S^{\alpha \beta}=N^{\alpha \beta}-\frac{1}{2} D(1-\mu) \epsilon^{\alpha \beta} B_{\lambda \mu} \bar{K}^{\lambda \mu}, \\
& \bar{T}^{\alpha \beta}=\bar{K}^{\alpha \beta}+\frac{1}{2} A(1+\nu) \epsilon^{\alpha \beta} B_{\lambda \mu} N^{\lambda \nu},
\end{aligned}
$$

we obtain a set of field equations identical to (2.38)-(2.41), (2.54) and (2.55).

The simplifications embodied in (3.4)-(3.7) have also been suggested by Koiter [14], although we feel that our arguments for adopting them are sounder than his.

4. Reduction of the simplified equations for arbitrary shells of non-zero Gaussian curvature. For shell of nonzero Gaussian curvature $(K \neq 0)$, we reduce our simplified field equations to four, coupled, second-order scalar equations. The method of reduction is similar to the one used by the author [5] to reduce the simplified Sanders' equations, and it is based in part on stress function representations used by Goldenveizer [15], Vekua [16], and Libai [4].

To begin the reduction, we observe that (2.42) can be identically satisfied by setting

where

$$
S^{\alpha}=\left.\epsilon^{\alpha \gamma} F\right|_{\gamma}
$$

$$
F=F^{\beta} a_{\beta}+F n
$$

is, for the moment, an arbitrary vector stress function. Using the component form of $\boldsymbol{S}^{\boldsymbol{\alpha}}$ given by (2.44) and the Gauss-Weingarten equations, (2.46), we find that

$$
\begin{aligned}
S^{\alpha \beta} & =\epsilon^{\alpha \gamma}\left(\left.F^{\beta}\right|_{\gamma}-F b_{\gamma}^{\beta}\right), \\
\left.D T\right|^{\alpha} & =-\epsilon^{\alpha \gamma}\left(F^{\beta} b_{\beta \gamma}+\left.F\right|_{\gamma}\right) .
\end{aligned}
$$

Rewriting (4.4) in the form

$$
b_{\lambda \beta} F^{\beta}=-\left.F\right|_{\lambda}-\left.D \epsilon_{\alpha \lambda} T\right|^{\alpha}
$$

and using the identity [4]

we obtain

$$
\bar{b}^{\lambda \mu} b_{\lambda \beta}=K \delta_{\beta}^{\mu}
$$

$$
F^{\beta}=K^{-1} \bar{b}^{\beta \lambda}\left(-\left.F\right|_{\lambda}+\left.D \epsilon_{\lambda \mu} T\right|^{\mu}\right) .
$$


Inserting (4.7) into (4.3), we arrive at a representation for $S^{\alpha \beta}$ in terms of $F$ and $T$ alone.

$$
S^{\alpha \beta}=\epsilon^{\gamma \alpha}\left[\left.\left(\left.K^{-1} b^{\beta \lambda} F\right|_{\lambda}\right)\right|_{\gamma}+F b_{\gamma}^{\beta}-\left.D \epsilon^{\beta \lambda}\left(\left.K^{-1} b_{\lambda}^{\mu} T\right|_{\mu}\right)\right|_{\gamma}\right] .
$$

In (4.8), and wherever possible, we use the fact that

$$
\epsilon_{\alpha \beta} b^{\beta \gamma}=\epsilon^{\beta \gamma} b_{\alpha \beta} .
$$

By the static-geometric analogy, $\bar{T}^{\alpha \beta}$ has the representation

$$
\bar{T}^{\alpha \beta}=\epsilon^{\gamma \alpha}\left[\left.\left(\left.K^{-1} \bar{b}^{\beta \lambda} G\right|_{\lambda}\right)\right|_{\gamma}+G b_{\gamma}^{\beta}+\left.A \epsilon^{\beta \lambda}\left(\left.K^{-1} b_{\lambda}^{\mu} S\right|_{\mu}\right)\right|_{\gamma}\right],
$$

where $G$ is the analogue of $F$.

Since $a_{\alpha \beta} S^{\alpha \beta}=S$ and $a_{\alpha \beta} \bar{T}^{\alpha \beta}=T$, it follows from (4.8)-(4.10) that

$$
\begin{aligned}
& S=\left.\epsilon^{\alpha \beta}\left(\left.K^{-1} b_{\alpha}^{\gamma} F\right|_{\beta}\right)\right|_{\gamma}+\left.D\left(\left.K^{-1} b^{\alpha \beta} T\right|_{\alpha}\right)\right|_{\beta} \\
& T=\left.\epsilon^{\alpha \beta}\left(\left.K^{-1} b_{\alpha}^{\gamma} G\right|_{\beta}\right)\right|_{\gamma}-\left.A\left(\left.K^{-1} b^{\alpha \beta} S\right|_{\alpha}\right)\right|_{\beta} .
\end{aligned}
$$

Two additional equations relating the four scalars $F, G, S$, and $T$ follow from (2.54), (2.55), and (4.8)-(4.10). With the aid of (4.6) and the identities

$$
B_{\alpha \beta} \epsilon^{\gamma \alpha}=H \delta_{\beta}^{\gamma}-b_{\beta}^{\gamma} \text { and } \epsilon^{\alpha \beta} b_{\alpha}^{\lambda} b_{\beta}^{\mu}=\epsilon^{\lambda \mu} K \text {, }
$$

there follows

$$
\begin{aligned}
&\left.\left(\left.K^{-1} \bar{b}^{\gamma \lambda} F\right|_{\lambda}\right)\right|_{\gamma}+2 H F-\left.D \epsilon^{\gamma \lambda}\left(\left.K^{-1} b_{\lambda}^{\mu} S\right|_{\mu}\right)\right|_{\gamma} \\
&= D(1-\mu)\left\{H\left[\left.\left(\left.K^{-1} \bar{b}^{\gamma \lambda} G\right|_{\lambda}\right)\right|_{\gamma}+2 H G+\left.A \epsilon^{\gamma \lambda}\left(\left.K^{-1} b_{\lambda}^{\mu} S\right|_{\mu}\right)\right|_{\gamma}\right]\right. \\
&\left.-\left[\nabla^{2} G-\left.\left.2 K^{-1} \bar{b}^{\beta \lambda} H\right|_{\beta} G\right|_{\lambda}+\left(4 H^{2}-2 K\right) G-\left.\left.2 A K^{-1} \epsilon^{\beta \lambda} b_{\lambda}^{\mu} H\right|_{\beta} S\right|_{\mu}\right]\right\}, \\
&\left.\left(\left.K^{-1} \bar{b}^{\gamma \lambda} G\right|_{\lambda}\right)\right|_{\gamma}+2 H G+\left.A \epsilon^{\gamma \lambda}\left(\left.K^{-1} b_{\lambda}^{\mu} S\right|_{\mu}\right)\right|_{\gamma} \\
&=-A(1+\nu)\left\{\left.H\left(\left.K^{-1} \bar{b}^{\gamma \lambda} F\right|_{\lambda}\right)\right|_{\gamma}+2 H F-\left.D \epsilon^{\gamma \lambda}\left(\left.K^{-1} b_{\lambda}^{\mu} T\right|_{\mu}\right)\right|_{\gamma}\right] \\
&\left.\quad-\left[\nabla^{2} F-\left.\left.2 K^{-1} \bar{b}^{\beta \lambda} H\right|_{\beta} F\right|_{\lambda}+\left(4 H^{2}-2 K\right) F+\left.\left.2 D K^{-1} \epsilon^{\beta \lambda} b_{\lambda}^{\mu} H\right|_{\beta} T\right|_{\mu}\right]\right\} .
\end{aligned}
$$

For a spherical shell with a midsurface of radius $R$ and outward unit normal $\boldsymbol{n}$,

$$
R b_{\alpha \beta}=-a_{\alpha \beta}, \quad \bar{b}_{\alpha \beta}=b_{\alpha \beta}, \quad R H=-1, \quad R^{2} K=1,
$$

and (4.11), (4.12), (4.14) and (4.15) reduce to

$$
\begin{gathered}
S=-D R^{-1} \Delta T \\
T=A R^{-1} \Delta S, \\
(\Delta+2) F=0, \\
(\Delta+2) G=0,
\end{gathered}
$$

where $\Delta=R^{2} \Delta^{2}$ is the Laplacian on the unit sphere. Setting

$$
\epsilon^{2}=A D / R^{2}=O\left(h^{2} / R^{2}\right),
$$

we may combine (4.17) and (4.18) into the single equation

$$
(\epsilon \Delta-i)\left[T+i(A / D)^{1 / 2} S\right]=0,
$$

which is somewhat simpler than Eq. (3.12) of [12], obtained using the unmodified stress-strain relations (2.5) and (2.6). 
5. The reduced equations for shells of zero mean curvature. If $H=0$, the reduced field equations derived in the preceding section simplify considerably. Noting that in this case $\bar{b}^{\alpha \beta}=-b^{\alpha \beta}$ and thus $\epsilon^{\alpha \beta} b_{\alpha}^{\gamma}=\epsilon^{\gamma \alpha} \bar{b}_{\alpha}^{\beta}=\epsilon^{\alpha \gamma} b_{\alpha}^{\beta}$, we find that (4.11), (4.12), (4.14) and (4.15) can be expressed in terms of $\nabla^{2}$ and the two operators

$$
L(F)=\left.\epsilon^{\alpha \beta}\left(\left.K^{-1} b_{\alpha}^{\gamma} F\right|_{\beta}\right)\right|_{\gamma}=\left.\epsilon^{\alpha \gamma}\left(\left.K^{-1} b_{\alpha}^{\beta} F\right|_{\beta}\right)\right|_{\gamma}
$$

and

$$
W(F)=-\left.\left(\left.K^{-1} b^{\alpha \beta} F\right|_{\alpha}\right)\right|_{\beta}
$$

as follows:

$$
\begin{gathered}
S=L(F)-D W\left(T^{\prime}\right), \\
T=L(G)+A W(S), \\
W(F)+D L(T)+D(1-\mu)\left(\nabla^{2}-2 K\right) G=0, \\
W(G)-A L(S)-A(1+\nu)\left(\nabla^{2}-2 K\right) F=0 .
\end{gathered}
$$

Although the pairs of equations (5.3)-(5.4) and (5.5)-(5.6) can each be written as a single equation for complex combinations of the unknowns, it is obvious that, unless $\mu=\nu=0$, the two sets of complex combinations will be different.

6. Catenoidal shells. In a right-hand cartesian reference frame with base vectors $i, j$, and $k$, the equation of a catenoid can be specified by

$$
x=R(\cosh \zeta \cos \theta i+\cosh \zeta \sin \theta j+\zeta \mathbf{k}) .
$$

With $\theta^{1}=\theta$ and $\theta^{2}=\zeta$, the components of the metric and curvature tensors become

$$
\begin{aligned}
& {\left[a_{\alpha \beta}\right]=R^{2} \cosh ^{2} \zeta\left[\begin{array}{ll}
1 & 0 \\
0 & 1
\end{array}\right],} \\
& {\left[b_{\alpha \beta}\right]=R\left[\begin{array}{rr}
-1 & 0 \\
0 & 1
\end{array}\right] .}
\end{aligned}
$$

Let

$$
S_{\theta}=S^{11}\left(a_{11} / a^{11}\right)^{1 / 2}, \text { etc. }
$$

denote the physical components of $S^{\alpha \beta}$, and let primes and dots denote differentiation with respect to $\zeta$ and $\theta$ respectively. The four equations implied by (4.8) take the form

$$
\begin{aligned}
R S_{\theta} & =\left(F^{\prime}+F \tanh \zeta\right)^{\circ}-D\left(T^{\prime \prime}+T \tanh \zeta\right) \\
R S_{\theta \zeta} & =-\left(F^{\prime \prime}+F^{\prime} \tanh \zeta+F \operatorname{sech}^{2} \zeta\right)-D\left(T^{\prime \prime}+T \tanh \zeta\right)^{\circ} \\
R S_{\zeta \theta} & =F^{\prime} \tanh \zeta-F \operatorname{sech}^{2} \zeta-F^{*}+D\left(T^{\prime \prime}+T \tanh \zeta\right)^{\circ} \\
R S_{\zeta} & =\left(F^{\prime}+F \tanh \zeta\right)^{\prime}+D\left(-T^{\prime} \tanh \zeta+T^{*}\right) .
\end{aligned}
$$

By :1se of the static-geometric analogy $\left(F \leftrightarrow G, S_{\theta} \leftrightarrow \bar{T}_{\theta}=T_{\zeta}\right.$ etc.) expressions for $i_{\theta}$, che, can be immediately written down, if needed.

The operators $\nabla^{2}-2 K, L$ and $W$ read

$$
R^{2}\left(\nabla^{2}-2 K\right) F=\operatorname{sech}^{2} \zeta\left(F^{\prime \prime}+F^{\prime \cdot}+F \operatorname{sech}^{2} \zeta\right),
$$




$$
\begin{gathered}
R L(F)=2\left(F^{\prime}+F \tanh \zeta\right)^{\circ} \\
R W(F)=F^{\prime \prime}+2 F^{\prime} \tanh \zeta-F^{\prime} .
\end{gathered}
$$

It is convenient to introduce the change of variable

$$
(F, G, S, T)=(\phi, \gamma, \sigma, \tau) \operatorname{sech} \zeta
$$

under which (6.5)-(6.11) go into

$$
\begin{aligned}
& R S_{\theta}=\operatorname{sech} \zeta\left[\phi^{\prime \prime}-D\left(\tau^{\prime \prime}-\tau^{\prime} \tanh \zeta-\tau \operatorname{sech}^{2} \zeta\right)\right], \\
& R S_{\theta \zeta}=-\operatorname{sech} \zeta\left(\phi^{\prime \prime}-\phi^{\prime} \tanh \zeta+D \tau^{\prime}\right), \\
& R S_{\zeta \theta}=\operatorname{sech} \zeta\left(\phi^{\prime} \tanh \zeta-\phi-\phi^{*}+D{\tau^{\prime}}^{\circ}\right) \text {, } \\
& R S_{\zeta}=\operatorname{sech} \zeta\left[\phi^{\prime}+D\left(-\tau^{\prime} \tanh \zeta+\tau \tanh ^{2} \zeta+\tau^{*}\right)\right], \\
& R^{2} \cosh \zeta\left(\nabla^{2}-2 K\right)(\phi \operatorname{sech} \zeta) \equiv \operatorname{sech}^{2} \zeta\left(\phi^{\prime \prime}-2 \phi^{\prime} \tanh \zeta+\phi^{*}+\phi\right) \\
& \equiv \operatorname{sech}^{2} \zeta \mathcal{D}(\phi) \text {, } \\
& R \cosh \zeta L(\phi \operatorname{sech} \zeta)=2 \phi^{\prime \prime}, \\
& R \cosh \zeta W(\phi \operatorname{sech} \zeta)=\phi^{\prime \prime}-\phi-\phi^{\cdot “} \equiv \mathscr{W}(\phi) \text {. }
\end{aligned}
$$

The governing equations (5.3)-(5.6) now read

$$
\begin{gathered}
R \sigma=2 \phi^{\prime}-D W(\tau), \\
R \tau=2 \gamma^{\prime}+A W(\sigma), \\
W(\phi)+2 D \tau^{\prime}+\underline{(D / R)(1-\mu) \operatorname{sech}^{2} \zeta D(\gamma)}=0, \\
W(\gamma)-2 A \sigma^{\prime}-\underline{(A / R)(1+\nu) \operatorname{sech}^{2} \zeta D(\phi)}=0 .
\end{gathered}
$$

In the derivation of his complex shell equations [7], Novozhilov assumes (in our notation) that $\epsilon_{\alpha \beta} S^{\alpha \beta}=\epsilon_{\alpha \beta} \bar{T}^{\alpha \beta}=0$. In particular, this assumption removes the underlined terms in the above equations, and this is why Novozhilov obtains constant coefficient equations for the catenoidal shell. It is obvious from the form of Eqs. (6.26) and (6.27) to follow that, in general, this simplification is not permissible.

By differential elimination of $\sigma$ and $\tau,(6.20)-(6.23)$ can be reduced to two coupled equations for $\phi$ and $\gamma$ :

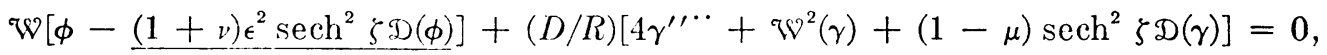

$\mathscr{W}\left[\gamma-(1-\mu) \epsilon^{2} \operatorname{sech}^{2} \zeta \mathscr{D}(\gamma)\right]-(A / R)\left[4 \phi^{\prime \prime} \cdot \cdot{ }^{\prime} \mathcal{W}^{2}(\phi)+(1+\nu) \operatorname{sech}^{2} \zeta D(\phi)\right]=0$,

where $\epsilon$ is defined by (4.21). The second, underlined term in each of the first brackets in (6.24) and (6.25) is $O\left(h^{2} / L^{2}\right)$ compared to the first term in the same bracket, where $L$ is the "characteristic wavelength" of the deformation pattern. Since the neglect of normal stress effects in classical shell theory introduces errors of $O\left(h^{2} / L^{2}\right)$ into the stressstrain relations [2], it should be possible to replace these equations by

$$
\Re(\phi)+(D / R)\left[4 \gamma^{\prime \prime}+W^{2}(\gamma)+(1-\mu) \operatorname{sech}^{2} \zeta D(\gamma)\right]=0,
$$




$$
W(\gamma)-(A / R)\left[4 \phi^{\prime \prime} \cdot W^{2}(\phi)+(1+\nu) \operatorname{sech}^{2} \zeta \mathcal{D}(\phi)\right]=0 .
$$

(Unfortunately, we are unable to give a more rigorous argument.) Even with this simplification, it is obvious, because of the presence of the factors $(1-\mu)$ and $(1+\nu)$, that (6.26) and (6.27) cannot be combined into a single complex equation.

Shallow shell theory. If $\zeta \gg 1$, the catenoid is shallow with respect to the base plane $\zeta=0$, and its meridional profile assumes a logarithmic shape. Within the range of validity of shallow shell theory, we may set $\operatorname{sech}^{2} \zeta=0$. The simplified equations so obtained may then be combined into the single equation

$$
\psi^{\prime \prime}-\psi-\psi^{\cdot}+i \epsilon\left(\psi^{\prime \prime \prime \prime}+2 \psi^{\prime \prime \cdot}+\psi^{\cdots \cdot}-2 \psi^{\prime \prime}+2 \psi^{\cdot \cdot}+\psi\right)=0
$$

where

$$
\psi=\gamma+i(A / D)^{1 / 2} \phi .
$$

The characteristic equation (6.28) agrees with that obtained by Lardner [17], who obtained this result by specializing the standard shallow shell equations.

Axisymmetric and simple sinusoidal stress states. It has been long known that the exact equations for shells of revolution undergoing axisymmetric deformation can be reduced to two coupled second-order equations. Actually, it is only necessary to require that the stresses be axisymmetric to accomplish this reduction-the displacement may be multivalued, of the dislocation type [18]. Novozhilov pointed out that if his simplified complex equations were applied to shells of revolution subject to wind-type ( $=$ simple sinusoidal $=$ lateral $=$ bending) loads, the conditions of overall force and moment equilibrium could be used to reduce these equations to a single, second-order, ordinary differential equation. Chernin [19] later showed how this reduction could be performed exactly starting from the full unreduced shell equations. He obtained two coupled, second-order equations, (32) and (33) of [19]. Wan [20] has reproduced Chernin's results using a slightly more general set of shell equations, and has included the possibility of dislocational solutions.

In view of these remarks, our reduced equations for the catenoidal shell should lead to a coupled second-order ordinary differential equation for axisymmetric and simple sinusoidal stress distributions. They do and therefore may be compared for aceuracy against the exact equations of Chernin [19] or Wan [20].

Consider first axisymmetric stress distributions. It is not difficult to show that $\phi$ and $\gamma$ must be of the form

$$
\begin{aligned}
& \phi=B_{1} \theta \sinh \zeta+\Phi(\zeta), \\
& \gamma=B_{2} \theta \sinh \zeta+\Gamma(\zeta) .
\end{aligned}
$$

The constants $B_{1}$ and $B_{2}$ can be directly related to the net axial force and its geometric, dislocation analogue. Inserting (6.30) and (6.31) into (6.22) and (6.23), we find that

$$
\Psi^{\prime \prime}-\Psi+i \beta \operatorname{sech}^{2} \zeta\left(\Psi^{\prime \prime}-2 \Psi^{\prime} \tanh \zeta+\Psi\right)=0
$$

wher:

$$
\Psi==\mathrm{\Gamma}+i\left(\frac{A(1+\nu)}{D(1-\mu)}\right)^{1 / 2} \Phi
$$

and

$$
\beta=\epsilon[(1-\mu)(1+\nu)]^{1 / 2} .
$$


By inspection, sinh $\zeta$ is a homogeneous solution of (6.32). Thus $\Psi$ is of the form

$$
\Psi=C_{1} \sinh \zeta+\sinh \zeta \Psi_{*}
$$

where

$$
\left(1+i \beta \operatorname{sech}^{2} \zeta\right) \Psi_{*}^{\prime}=C_{2} \operatorname{csch}^{2} \zeta
$$

The $\sinh \zeta$ solution is a null stress solution; hence we may set $C_{1}=0$. The complex constant $C_{2}$ may be directly related to the net axial torque and its geometric dislocational analogue.

Returning to (6.20) and (6.21), we have particular solutions of the form

$$
\sigma_{p}=2 B_{1} R^{-1} \cosh \zeta, \quad \tau_{p}=2 B_{2} R^{-1} \cosh \zeta
$$

plus homogeneous solutions which satisfy

$$
\left[\epsilon\left(d^{2} / d \zeta^{2}-\underline{1}\right)-i\right]\left(\tau_{h}+i(A / D)^{1 / 2} \sigma_{h}\right)=0 .
$$

This equation is simpler than the corresponding two real-valued equations (for different variables) one obtains by specializing Reissner's equations [18], [21], which, as they stand, are neither constant coefficient nor can be combined into a single complex equation. However this apparent discrepancy is resolved by noting that both sets of homogeneous equations are of the boundary layer type, so that the underlined term in (6.38) and the analogous type of term in Reissner's equations make a uniformly small contribution of relative order $O(h / R)$ to the solutions, and hence may be neglected.

Now consider stress distributions proportional to $\sin \theta$. Again, it is not difficult to show that $\phi$ and $\gamma$ must be of the form

$$
\begin{aligned}
& \phi=B_{3} \theta \sin \theta+f(\zeta) \cos \theta \\
& \gamma=B_{4} \theta \sin \theta+g(\zeta) \cos \theta .
\end{aligned}
$$

The constants $B_{3}$ and $B_{4}$ can be related to the net shear force over any circular edge and its geometric, dislocational analogue. Inserting (6.39) and (6.40) into (6.22) and (6.23) and setting

$$
\sigma=s(\zeta) \sin \theta, \quad \tau=t(\zeta) \sin \theta
$$

we have

$$
\begin{aligned}
& f^{\prime \prime}+2 D t^{\prime}+(D / R)(1-\mu) \operatorname{sech}^{2} \zeta\left(g^{\prime \prime}-2 g^{\prime} \tanh \zeta\right)=2 B_{3}, \\
& g^{\prime \prime}-2 A s^{\prime}-(A / R)(1+\nu) \operatorname{sech}^{2} \zeta\left(f^{\prime \prime}-2 f^{\prime} \tanh \zeta\right)=2 B_{4} .
\end{aligned}
$$

These equations may be immediately integrated once:

$$
\begin{aligned}
& f^{\prime}+2 D t+(D / R)(1-\mu) g^{\prime} \operatorname{sech}^{2} \zeta=2 B_{3} \zeta+B_{5}, \\
& g^{\prime}-2 A s-(A / R)(1+\nu) f^{\prime} \operatorname{sech}^{2} \zeta=2 B_{4} \zeta+B_{6} .
\end{aligned}
$$

The constants $B_{5}$ and $B_{6}$ may be related to the net moment acting over any circular edge and its geometric, dislocational analogue. Solving these two equations algebraically for $f^{\prime}$ and $g^{\prime}$, inserting the results along with (6.41) and (6.42) into (6.20) and (6.21), and neglecting terms of order $h^{2} / R^{2}$ compared to unity in the final equations, we get

$$
R s=-D\left(t^{\prime \prime}-4 t\right)-4 B_{3} \zeta-2 B_{5}+2(D / R)(1-\mu) \operatorname{sech}^{2} \zeta\left(2 B_{4} \zeta+B_{6}\right),
$$




$$
R t=A\left(s^{\prime \prime}-4 s\right)-4 B_{4} \zeta-2 B_{6}-2(A / R)(1+\nu) \operatorname{sech}^{2} \zeta\left(2 B_{3} \zeta+B_{5}\right) .
$$

The homogeneous form of (6.47) and (6.48), which can be conveniently combined into the single equation

$$
\left[\epsilon\left(d^{2} / d^{2} \zeta-\underline{4}\right)-i\right]\left(t_{h}+i(A / D)^{1 / 2} s_{h}\right)=0,
$$

is somewhat simpler than the corresponding two real homogeneous characteristic equations (for different variables) that follow upon specializing Wan's equations ((5.1) and (5.2) in [20]) to catenoidal shells. However, as with the case of axisymmetric stresses, we resolve this discrepency by observing that both our Eq. (6.49) and Wan's are of the boundary layer type so that the underlined term in (6.49) and the analogous terms in (5.1) and (5.2) of [20] can be neglected, bringing the two results into agreement.

7. Helicoidal shells. In a right-handed cartesian reference frame with base vectors $i, j$ and $k$, the equation of a helicoid can be specified by

$$
x=R(\sinh \zeta \cos \theta i+\sinh \zeta \sinh \theta j+\theta k) .
$$

(Here, we follow Koiter [14] and use $\zeta$ instead of $r=R \sinh \zeta$ as one of the surface coordinates. This choice seem to condense considerably a number of the equations which follow.) With $\theta^{1}=\zeta$ and $\theta^{2}=\theta$, the components of the metric and curvature tensors become

$$
\begin{aligned}
& {\left[a_{\alpha \beta}\right]=R^{2} \cosh ^{2} \zeta\left[\begin{array}{ll}
1 & 0 \\
0 & 1
\end{array}\right],} \\
& {\left[b_{\alpha \beta}\right]=-R\left[\begin{array}{ll}
0 & 1 \\
1 & 0
\end{array}\right] .}
\end{aligned}
$$

Let

$$
S_{5}=S^{11}\left(a_{11} / a^{11}\right)^{1 / 2}, \text { etc., }
$$

denote the physical components of $S^{\alpha \beta}$, and let primes and dots denote differentiation with respect to $\zeta$ and $\theta$ respectively. The four equations implied by (4.8) take the form

$$
\begin{aligned}
R S_{\zeta} & =-F^{\prime} \tanh \zeta+F \operatorname{sech}^{2} \zeta+F^{\prime}+D\left(T^{\prime}+T \tanh \zeta\right)^{\circ} \\
R S_{\zeta \theta} & =\left(F^{\prime}+F \tanh \zeta\right)^{\circ}+D\left(T^{\prime \prime} \tanh \zeta-T^{\prime}\right), \\
R S_{\theta \zeta} & =-\left(F^{\prime}+F \tanh \zeta\right)^{\circ}-D\left(T^{\prime \prime}+T^{\prime} \tanh \zeta\right), \\
R S_{\theta} & =-\left(F^{\prime \prime}+F^{\prime} \tanh \zeta+F \operatorname{sech}^{2} \zeta\right)+D\left(T^{\prime}+T \tanh \zeta\right)^{\circ}
\end{aligned}
$$

By use of the static-geometric analogy, expressions for $T_{\theta}$, etc., may be immediately written down, if needed.

The operators $\nabla^{2}-2 K, L$ and $W$ read

$$
\begin{gathered}
R^{2}\left(\nabla^{2}-2 K\right) F=\operatorname{sech}^{2} \zeta\left(F^{\prime \prime}+F^{\prime}+F \operatorname{sech}^{2} \zeta\right), \\
R L(F)=-F^{\prime \prime}-2 F^{\prime \prime} \tanh \zeta+F^{*} \\
R W\left(F^{\prime}\right)=-2\left(F^{\prime}+F^{\prime} \tanh \zeta\right)^{\circ} .
\end{gathered}
$$

It is convenient to introduce the change of variables

$$
(F, G, S, T)=(\phi, \gamma, \sigma, \tau) \operatorname{sech} \zeta
$$


under which (7.5)-(7.11) go into

$$
\begin{aligned}
& R S_{\zeta}=\operatorname{sech} \zeta\left(-\phi^{\prime} \tanh \zeta+\phi+\phi^{*}+D{\tau^{\prime}}^{\circ}\right), \\
& R S_{\zeta \theta}=\operatorname{sech} \zeta\left[\phi^{\prime}+D\left(\tau^{\prime} \tanh \zeta-\tau \tanh ^{2} \zeta-{\tau^{\prime}}^{\prime}\right)\right] \text {, } \\
& R S_{\theta \zeta}=-\operatorname{sech} \zeta\left[\phi^{\prime}+D\left(\tau^{\prime \prime}-\tau^{\prime} \tanh \zeta-\tau \operatorname{sech}^{2} \zeta\right)\right] \text {, } \\
& R S_{\theta}=-\operatorname{sech} \zeta\left(\phi^{\prime \prime}-\phi^{\prime} \tanh \zeta-D{\tau^{\prime}}^{\circ}\right), \\
& R^{2} \cosh \zeta\left(\nabla^{2}-2 K\right)(\phi \operatorname{sech} \zeta)=\operatorname{sech}^{2} \zeta\left(\phi^{\prime \prime}-2 \phi^{\prime} \tanh \zeta+\phi^{\prime \prime}+\phi\right) \\
& \equiv \operatorname{sech}^{2} \zeta \mathscr{D}(\phi), \\
& R \cosh \zeta L(\phi \operatorname{sech} \zeta)=-\left(\phi^{\prime \prime}-\phi-\phi^{*}\right) \\
& \equiv-\mathfrak{L}(\phi), \\
& R \cosh \zeta W(\phi \operatorname{sech} \zeta)=-2 \phi^{\prime} .
\end{aligned}
$$

The basic, reduced Eqs. (5.3)-(5.6) now read

$$
\begin{gathered}
R \sigma=-\mathscr{L}(\phi)+2 D{\tau^{\prime}}^{\prime}, \\
R \tau=-\mathscr{L}(\gamma)-2 A{\sigma^{\prime}}^{\prime}, \\
2{\phi^{\prime}}^{\prime}+D \mathscr{L}(\tau)-(D / R)(1-\mu) \operatorname{sech}^{2} \zeta D(\gamma)=0, \\
2 \gamma^{\prime}-A \mathscr{L}(\sigma)+(A / R)(1+\nu) \operatorname{sech}^{2} \zeta D(\phi)=0 .
\end{gathered}
$$

By differential elimination of $\sigma$ and $\tau,(7.20)-(7.23)$ can be reduced to the following two coupled equations for $\phi$ and $\gamma$ :

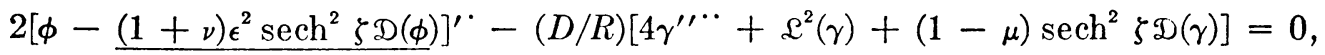

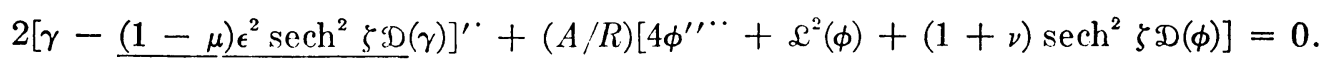

As with the catenoidal shell, the second (underlined) terms in each of the first brackets in (7.24) and (7.25) are $O\left(h^{2} / L^{2}\right)$ compared to the first terms in each of these brackets, so that we should be able to replace these equations by the simpler ones

$$
\begin{aligned}
& 2 \phi^{\prime}-(D / R)\left[4 \gamma^{\prime \prime}+\mathscr{L}^{2}(\gamma)+(1-\mu) \operatorname{sech}^{2} \zeta \mathcal{D}(\gamma)\right]=0, \\
& 2 \gamma^{\prime}+(A / R)\left[4 \phi^{\prime \prime} \cdot \cdot \mathscr{L}^{2}(\phi)+(1+\nu) \operatorname{sech}^{2} \zeta \mathcal{D}(\phi)\right]=0 .
\end{aligned}
$$

It is obvious, because of the presence of the factors $(1-\mu)$ and $(1+\nu)$, that $(7.26)$ and (7.27) cannot be combined into a single complex equation.

Shallow shell theory. If $\zeta \gg 1$, the helicoidal shell becomes shallow with respect to the plane of the base vectors $i$ and $j$. Within the range of validity of shallow shell theory, we may set $\operatorname{sech}^{2} \zeta=0$ in (7.26) and (7.27). The simplified equations so obtained may be combined into the single equation

$$
\epsilon\left(\psi^{\prime \prime \prime \prime}+2 \psi^{\prime \prime}+\psi^{\cdots}-2 \psi^{\prime \prime}+2 \psi^{*}+\psi\right)+2 i \psi^{\prime}=0
$$

where

$$
\psi=\gamma+i(A / D)^{1 / 2} \phi
$$


It is not difficult to verify that the characteristic equation (7.28) agrees with that obtained directly from shallow shell theory using polar coordinates $(r, \theta)$, provided one sets $r=\frac{1}{2} \exp (\zeta)$.

Axisymmetric and simple sinusoidal stress states. Helicoidal shells, like shells of revolution, admit special classes of deformation in which the resulting states of stress and strain are either asymmetric or proportional to $\sin \theta$ or $\cos \theta$. Furthermore, in the axisymmetric case, overall equilibrium and compatibility conditions imply the existence of first and second integrals of each of the resulting ordinary differential equations. However, Professor Wan has pointed out (in a private communication) that in the sinusoidal case these conditions imply the existence of only a first integral of each of the resulting ordinary differential equations.

Axisymmetric stress problems in helicoidal shells have been extensively studied by Reissner and his students [22]-[27]. Our equations lead to a second-order differential equation which agrees exactly with that obtained by Cohen [28], Reissner [25], and others. Wan [29], [30], [31] has considered several other problems which produce simple sinusoidal stress distributions, but, except for shallow shells of revolution, he has not derived the reduced governing differential equations. We show that such problems may be reduced to the solution of a coupled set of third-order differential equations.

For axisymmetric stresses, it is not difficult to show that $\phi$ and $\gamma$ must be of the form

$$
\begin{aligned}
& \phi=B_{1} \theta \sinh \zeta+\phi(\zeta) \\
& \gamma=B_{3} \theta \sinh \zeta+\Gamma(\zeta) .
\end{aligned}
$$

The constants $B_{1}$ and $B_{2}$ can be related, respectively, to the net axial force along an edge $\zeta=$ const. and its geometric analogue. Inserting (7.30) and (7.31) into (7.22) and (7.23) and using (7.20) and (7.21) to express $\sigma$ and $\tau$ in terms to $\phi$ and $\gamma$, we obtain $(D / R)\left[\Gamma^{\prime \prime \prime \prime}-2 \Gamma^{\prime \prime}+\Gamma+(1-\mu) \operatorname{sech}^{2} \zeta\left(\Gamma^{\prime \prime}-2 \Gamma^{\prime} \tanh \zeta+\Gamma\right)\right]=2 B_{1} \cosh \zeta$

$(A / R)\left[\Phi^{\prime \prime \prime \prime}-2 \Phi^{\prime \prime}+\Phi+(1+\nu) \operatorname{sech}^{2} \zeta\left(\Phi^{\prime \prime}-2 \Phi^{\prime} \tanh \zeta+\Phi\right)\right]=-2 B_{1} \cosh \zeta$.

Because these equations are uncoupled and of identical form, it is sufficient to consider just the first. By inspection $\sinh \zeta$ is a homogeneous solution of (7.32). Hence the general solution of this equation is of the form

$$
\Gamma=C_{1} \sinh \zeta+\sinh \zeta \int \Gamma_{*} d \zeta .
$$

Since $\sinh \zeta$ is a null-stress solution, we can set $C_{1}=0$. Substituting this expression into (7.32), we obtain an equation for $\Gamma_{*}$ which may be expressed as a total differential. Integrating this, we obtain, finally, the following second-order differential equation for $\Gamma_{*}$ :

$$
\begin{array}{r}
(D / R)\left[\Gamma_{a *}^{\prime \prime} \sinh ^{2} \zeta+2 \Gamma_{*}^{\prime} \sinh \zeta \cosh \zeta-2 \Gamma_{*}+(1-\mu) \Gamma_{*} \tanh ^{2} \zeta\right] \\
=B_{1} \cosh ^{2} \zeta+B_{3} .
\end{array}
$$

The constant $B_{3}$ may be rclated to a combination of the net axial force and the net torque per unit winding acting on an edge $\zeta=$ const. [25]. This equation may be shown to agree with Eq. (26) of [25) providing that in the latter equation we use Eq. (34) of [25] to express $\beta_{r}$ in terms of $\Psi$, and then set $r=R \sinh \zeta$ and $\psi=\tanh \zeta \int \Gamma_{*} d \zeta$. 
For stress distribution proportional to $\sin \theta$ or $\cos \theta$, it is not difficult to show that $\phi$ and $\gamma$ must be of the form

$$
\begin{aligned}
& \phi=B_{5} \theta \cos \theta+f(\zeta) \sin \theta, \\
& \gamma=B_{6} \theta \sin \theta+g(\zeta) \cos \theta .
\end{aligned}
$$

The constants $B_{6}$ and $B_{5}$ can be related, respectively, to the net moment across an edge $\theta=$ const. and its geometric analogue [29]. Inserting (7.36) and (7.37) into (7.26) and (7.27), we find that the resulting equations can be written as total differentials. (This is also true of (7.24) and (7.25) with the underlined terms retained.) Integrating once we obtain the following two coupled third-order equations for $f$ and $g$ :

$$
\begin{aligned}
& 2 f-(D / R)\left[g^{\prime \prime \prime}-4 g^{\prime}+(1-\mu) g^{\prime} \operatorname{sech}^{2} \zeta\right]=2(D / R)(1-\mu) B_{5} \tanh \zeta+B_{7}, \\
& -2 g+(A / R)\left[f^{\prime \prime \prime}-4 f^{\prime}+(1+\nu) f^{\prime} \operatorname{sech}^{2} \zeta\right]=2(A / R)(1+\nu) B_{6} \tanh \zeta+B_{8},
\end{aligned}
$$

where $B_{7}$ and $B_{8}$ are arbitrary constants of integration.

Finally, it can be shown that stress distributions of the type

$$
\sigma=s(\zeta) \theta \cos \theta, \quad \tau=t(\zeta) \theta \sin \theta
$$

lead to a coupled set of fourth-order equations, which again can each be integrated once. Stress distributions of this type arise, for example, in the beamlike bending of a helicoidal shell subject to end shears and moments. Wan has recently considered such problems for shallow helicoidal shells [32].

Acknowledgment. I wish to thank Profs. Rcissner and Wan, each of whom sent me detailed criticisms of the manuscript.

\section{BIBLIOGRAPHY}

1. J. L. Sanders, Jr., An improved first approximation theory for thin shells, N.A.S.A. liept. no. 24, 1959.

2. W. T. Koitcr, $A$ consistant first approximation in the general thcory of thin elastic shells, Proc. Sympos. Thin Elastic Shells, North-Holland, Ansterdam, 1966), pp. 12-33

3. B. Budiansky" and J. L. Sanders, Jr., On the "best" first-order linear shell theory, Progress in Applied Mechanics, MacNillan and Co., New York, 1963, pp. 129-140

4. A. Lihai, Invariant stress and deformation functions for doubly curved shells, J. Appl. Mech. 34, 4348(1967).

5. J. G. Simmonds, Further reduction of equation (20) for artitrary shells of non-zero Gaussian curvature (Appendix to [6]), Proc. Second Sympos. Thin Elastic Shells, Springer-Verlag, Berlin and New York, 1969, pp. 157-160

6. J. L. Sanders, Jr., On the shell equations in complex form, Proc. Second Sympos. Thin Elastic Shells, Springer-Verlag, Berlin and New York, 1969, pp. 135-156

7. V. V. Novozhilov, Thin shell theory, linglish translation, 2nd edition, Noordhoff, Groningen, 1965

8. W. T. Koiter, A systematic simplification of the general equations in the linear theory of thin shells Proc. Nederl. Akad. Wetensch. B64, 612-619 (1961)

9. A. L. Goldenveizer, Equalions of the theory of shells in displacements and stress functions, Prikl. Mat. Mekh. 21, 801-814 (1950); (English translation Aztec School of Languages, West Acton, Massachuset.s, Document \#A2T-63-294-RUL., avialable from M.I.T. Lincoln Laboratory)

10. F. Y. M. Wan, The exact reduction of equations of elastic shells of revolution, Studies in Appl. Math. $48,361-375)(1969)$

11. W. T. Koiter, A spherical shell under point loals of its poles, Progress in Applied Mechanics, MacMillan, New York, 1963, pp. 15j-169

12. J. G. Simmonds, Green's functions for closed elastic spherical shells: exuct and accurate approximate solutions, Nederl. Akad. Wetensch. Proc. B71, 236-249 (1965)

13. J. G. Simmonds, $A$ set of simple, accurate equations for circular cylindrical elastic shells, Int. J. Solids and Structures 2, 523-541 (1966) 
14. W. T. Koiter, A comparison between John's refined interior shell equations and classical shell theory Z.A.M.P. 20, 642-652 (1969)

15. A. L. Goldenveizer, Theory of elastic thin shells, GITTL, Moscow, 1953; english transl., Pergamon Press, New York, 1961

16. I. N. Vekua, Generalized analytic functions, Fizmatgiz, Moscow, 1959; transl., Pergamon Press, New York 1962, Chapt. VI.

17. T. J. Lardner, On the stress distribution in a shallow logarithmic shell of revolution, J. Math. and Physics 45, 23-34 (1966)

18. E. Reissner and F. Y. M. Wan, Rotationally symmetric stress and strain in shells of revolution, Studies Appl. Math. 48, 1-17 (1969)

19. V.S. Chernin, On the system of differential equations of equilibrium of shells of revolution under bending loads (English translation) P.M.M., 23, 258-265 (1959); J. Appl. Math. Mech. 23, 372-382 (1959)

20. F. Y. M. Wan, Circumferentially sinusoidal stress and strain in shells of revolution, to appear in Int. J. Solids and Structures.

21. E. Reissner, On the equations for finite symmetrical deflections of thin shells of revolution, Progress in Applied Mechanics, MacMillan, New York, 1963, pp. 171-178

22. E. Reissner, Small rotationally symmetric deformations of shallow helicoidal shells, J. Appl. Mech. 22, 31-34 (1955)

23. J. K. Knowles and E. Reissner, Torsion and extension of helicoidal shells, Quart. Appl. Math. 17, 409-422 (1960)

24. E. Reissner, On twisting and stretching of helicoidal shells, Proc. Symp. Thin Elastic Shells, North Holland, Amsterdam, 1960, pp. 434-466

25. E. Reissner, Note on axially symmetric stress distributions in helicoidal shells, Miszellaneen der Angewandten Mechanik (Tollmien Festschrift), Akademie-Verlag, Berlin 1962, pp. 257-266

26. D. P. O'Mathuna, Rotationally symmetric deformations in helicoidal shells, J. Math and Physics 42, 100-133 (1963)

27. E. Reissner and F. Y. M. Wan, On axial extension and torsion of helicoidal shells, J. Math and Phys. 47, 1-31 (1968)

28. J. W. Cohen, The inadlequacy of the classical stress-strain relations for the right helicoidal shell, Proc. Sympos. Thin Elastic Shells, North Holland, Amsterdam, 1960, pp. 415-433

29. F. Y. M. Wan, The side-force problems for shallow helicoidal shells, J. Appl. Mech. 36, 292-295 (1969)

30. F. Y. M. Wan, Pure bending of shallow helicoidal shells, J. Appl. Mech. 35, 387-392 (1968)

31. F. Y. M. Wan, A class of unsymmetric stress distributions in helicoidal shells, Quart. Appl. Math. 24, 374-379 (1967)

32. F. Y. M. Wan, St. Venant flexure of pretwisted rectangular plates, Int. J. Engr. Sci. 7, 351-360 (1969) 